\title{
Expect the Unexpected: SGLT-2 \\ Inhibitors in the Treatment of Type 2 Diabetes and/or Heart Failure
}

\author{
Jelena P. Seferovic ${ }^{a}$ Petar M. Seferovic ${ }^{b, c}$ \\ ${ }^{a}$ Clinic for Endocrinology, Diabetes and Metabolic Disorders, Clinical Center Serbia and Faculty of Medicine, \\ University of Belgrade, Belgrade, Serbia; ${ }^{b}$ University of Belgrade Faculty of Medicine, Belgrade, Serbia; \\ 'Serbian Academy of Sciences and Arts, Belgrade, Serbia
}

In the review, Santos-Ferreira et al. [1] discuss different aspects of the association between two frequent comorbidities, type 2 diabetes (T2D) and heart failure (HF). There is an abundance of data suggesting that patients with T2D and HF have an increased risk of cardiovascular (CV) and all-cause mortality, as well as reduced quality of life $[2,3]$. While guidelines for individual treatments of T2D and HF are well established, recent findings from $\mathrm{CV}$ trials investigating glucose-lowering agents in T2D patients have revealed improved outcomes when treatments for both conditions are concurrently optimized.

Recently, few novel classes of glucose-lowering agents have been introduced. The results from the studies with sodium-glucose cotransporter 2 (SGLT2) inhibitors - a novel class of agents that inhibit glucose reabsorption in the proximal renal tubule [4] -yielded the greatest surprise. These agents are the main focus of the review article by Santos-Ferreira et al. [1], where three completed large CV outcome trials investigating SGLT2 inhibitors in patients with T2D and established atherosclerotic CV disease or multiple risk factors were elaborated in detail. The first one of these studies, EMPA-REG OUTCOME (Empagliflozin Cardiovascular Outcome Event Trial in Type 2 Diabetes Mellitus Patients), investigated the effects of

karger@karger.com

(c) 2020 S. Karger AG, Basel

www.karger.com/crd

Karger empagliflozin on CV outcomes in patients with T2D and established atherosclerotic disease [5] and found a 35\% relative risk reduction in the exploratory endpoint of $\mathrm{HF}$ hospitalization. A similar effect was observed in the CANVAS study program (integrated data from 2 trials, CANVAS [Canagliflozin Cardiovascular Assessment Study] and CANVAS-R [Canagliflozin Cardiovascular Assessment Study - Renal]) [6], where canagliflozin significantly reduced the exploratory endpoint of HF hospitalization versus placebo in patients with and without history of HF. A post-hoc analysis found that canagliflozin reduced the overall risk of HF events, with no clear difference in effects on HF with reduced ejection fraction (HFrEF) versus HF with preserved EF (HFpEF) events [7]. In the DECLARE-TIMI 58 (Dapagliflozin Effect on Cardiovascular Events-Thrombolysis in Myocardial Infarction 58) trial, dapagliflozin significantly reduced the risk of composite outcome of HF hospitalization or CV death versus placebo, and this was driven by a lower rate in HF hospitalization. This benefit was consistent in patients with and without recognized atherosclerotic disease, as well as in those with and without a prior HF history [8].

A meta-analysis of above-mentioned three trials confirmed individual findings, demonstrating consis- 
tent beneficial effect of SGLT2 inhibitors on the reduction of HF hospitalization by $31 \%$, regardless of atherosclerotic disease or prior history of HF [9]. Similar data was found in the real-world studies CVD-REAL (Comparative Effectiveness of Cardiovascular Outcomes in New Users of SGLT-2 Inhibitors) [10] and the ongoing EMPRISE (Non-Interventional Study on the Effectiveness and Safety of Empagliflozin Compared with DPP4 Inhibitors in Patients with Type 2 Diabetes in the United States) [11] trial, confirming the beneficial effect of SGLT-2 inhibitors on the prevention of HF hospitalization in patients with T2D, independent of atherosclerotic disease.

As noted in the review [1], the data from all these trials with SGLT2 inhibitors have changed the current understanding of the treatment paradigms of T2D and CV diseases, and HF in particular. Empagliflozin, as first-inclass SGLT2 inhibitor to demonstrate the beneficial effect on HF hospitalization, was recommended in the 2016 ESC guidelines for the diagnosis and treatment of HF [12] and Guidelines for CV prevention [13], for HF onset delay among patients with T2D. The recommendation was extended to other SGLT-2 inhibitors in the 2019 expert consensus report from the ESC Heart Failure Association [14]. In 2018, the consensus statement of the American Diabetes Association and European Association for the Study of Diabetes (ADA/EASD) included SGLT-2 inhibitors as the preferred treatment of T2D in patients with known HF or at risk of HF [15]. In addition, SGLT-2 inhibitors have been recommended as a second-line therapy, after metformin, or in patients in whom metformin is contraindicated or not tolerated.

Prior published data hold promise that SGLT-2 inhibitors have favorable effect in the prevention of HF among patients with $\mathrm{T} 2 \mathrm{D}$, across a wide spectrum of $\mathrm{CV}$ risk. However, whether SGLT-2 inhibitors could be beneficial in the treatment of HF, with or without T2D, is currently being investigated in several trials. The first completed trial, DAPA-HF (Dapagliflozin in Patients with Heart Failure and Reduced Ejection Fraction), reported a significant $26 \%$ risk reduction in the primary endpoint of $\mathrm{CV}$ mortality/HF hospitalization/urgent HF visit, in patients with HFrEF (LVEF $\leq 40 \%)$ and elevated natriuretic peptides, with similar results in patients with and without T2D [16]. Ongoing clinical trials, EMPEROR-Reduced ([Empagliflozin Outcome Trial in Patients with Chronic Heart Failure with Reduced Ejection Fraction]), DELIVER ([Dapagliflozin Evaluation to Improve the Lives of Patients with Preserved Ejection Fraction Heart Failure], and EMPEROR-Preserved [Empagliflozin Outcome Tri- al in Patients with Chronic Heart Failure with Preserved Ejection Fraction]), will confirm the potential benefit of SGLT2 inhibitors in patients with HFpEF and/or HFrEF, irrespective of T2D status.

Overall, in trials reported thus far, SGLT2 inhibitors have been well tolerated. Genital mycotic infections, most frequently reported adverse event, are usually mild and could be effectively treated $[5,6]$. Diabetic ketoacidosis may rarely occur, usually in patients with concomitant insulin therapy. However, reinitiating SGLT-2 inhibitors following ketoacidosis is not recommended because of an increased risk of recurrence $[5,6]$. In the CANVAS Program, a greater risk of bone fractures and lower limb amputations was seen with canagliflozin compared to placebo. The risk was higher in patients with previous amputations or peripheral arterial disease [6]. However, this was not seen with other SGLT2 inhibitors. Furthermore, a meta-analysis of the three large CV outcome trials suggested that an increased risk of these events was evident only in the CANVAS trial. Further research is warranted to determine if the observed risk is associated with SGLT2 inhibitor treatment and, if so, whether this is a class or individual agent effect. In DAPA-HF, among the highrisk HFrEF patients with or without T2D, no significant excess in serious adverse events was noted with dapagliflozin versus placebo [16].

In conclusion, the review "SGLT 2 Inhibitors in Heart Failure and Type 2 Diabetes: Hitting Two Birds with One Stone?" [1] provides relevant and accurate overview of existing data on SGLT2 inhibitors as novel class of glucose-lowering agents with convincing evidence in the prevention of $\mathrm{HF}$ in patients with $\mathrm{T} 2 \mathrm{D}$. Current guidelines for the treatment of T2D suggest that SGLT2 inhibitors should be used as second-line agents for glucose reduction and CV protection, after metformin. The first trial of patients with prevalent HF found that the risk of worsening $\mathrm{HF}$ or death from $\mathrm{CV}$ causes was lower in those who received dapagliflozin compared to placebo, regardless of the presence of T2D. Ongoing clinical trials will investigate the potential benefit of SGLT2 inhibitors in patients with both HFpEF and HFrEF, with or without T2D.

\section{Disclosure Statement}

The authors declare that they have no conflicts of interest to disclose. 


\section{References}

1 Santos-Ferreira D, Gonçalves-Teixeira P, Fontes-Carvalho R. SGLT-2 Inhibitors in Heart Failure and Type-2 Diabetes: Hitting Two Birds with One Stone? Cardiology. DOI: $10.1159 / 504694$.

2 Seferović PM, Petrie MC, Filippatos GS, Anker SD, Rosano G, Bauersachs J, et al. Type 2 diabetes mellitus and heart failure: a position statement from the Heart Failure Association of the European Society of Cardiology. Eur J Heart Fail. 2018 May;20(5):853-72.

3 MacDonald MR, Petrie MC, Varyani F, Ostergren J, Michelson EL, Young JB, et al.; CHARM Investigators. Impact of diabetes on outcomes in patients with low and preserved ejection fraction heart failure: an analysis of the Candesartan in Heart failure: Assessment of Reduction in Mortality and morbidity (CHARM) programme. Eur Heart J. 2008 Jun;29(11):1377-85.

4 Nauck MA. Update on developments with SGLT2 inhibitors in the management of type 2 diabetes. Drug Des Devel Ther. 2014 Sep;8: 1335-80.

5 Zinman B, Wanner C, Lachin JM, Fitchett D, Bluhmki E, Hantel S, et al.; EMPA-REG OUTCOME Investigators. Empagliflozin, cardiovascular outcomes, and mortality in type 2 diabetes. N Engl J Med. 2015 Nov; 373(22):2117-28.

6 Neal B, Perkovic V, Mahaffey KW, de Zeeuw D, Fulcher G, Erondu N, et al.; CANVAS Program Collaborative Group. Canagliflozin and cardiovascular and renal events in type 2 diabetes. N Engl J Med. 2017 Aug;377(7):644-57.

7 Figtree GA, Rådholm K, Barrett TD, Perkovic V, Mahaffey KW, de Zeeuw D, et al. Effects of canagliflozin on heart failure outcomes associated with preserved and reduced ejection fraction in type 2 diabetes mellitus. Circulation. 2019 May;139(22):2591-3.

8 Wiviott SD, Raz I, Bonaca MP, Mosenzon O, Kato ET, Cahn A, et al.; DECLARE-TIMI 58 Investigators. Dapagliflozin and cardiovascular outcomes in type 2 diabetes. N Engl J Med. 2019 Jan;380(4):347-57.

9 Zelniker TA, Wiviott SD, Raz I, Im K, Goodrich EL, Bonaca MP, et al. SGLT2 inhibitors for primary and secondary prevention of cardiovascular and renal outcomes in type 2 diabetes: a systematic review and meta-analysis of cardiovascular outcome trials. Lancet. 2019 Jan;393(10166):31-9.

10 Kosiborod M, Cavender MA, Fu AZ, Wilding JP, Khunti K, Holl RW, et al; CVD-REAL Investigators and Study Group*. Lower Risk of Heart Failure and Death in Patients Initiated on Sodium-Glucose Cotransporter-2 Inhibitors Versus Other Glucose-Lowering Drugs: The CVD-REAL Study (Comparative Effectiveness of Cardiovascular Outcomes in New Users of Sodium-Glucose Cotransporter-2 Inhibitors). Circulation. 2017 Jul;136(3):24959.

11 Patorno E, Pawar A, Franklin JM, Najafzadeh M, Déruaz-Luyet A, Brodovicz KG, et al. Empagliflozin and the Risk of Heart Failure Hospitalization in Routine Clinical Care. Circulation. 2019 Jun;139(25):2822-30.

12 Ponikowski P, Voors AA, Anker SD, Bueno H, Cleland JG, Coats AJ, et al.; Authors/Task Force Members; Document Reviewers. 2016 ESC Guidelines for the diagnosis and treatment of acute and chronic heart failure: the Task Force for the diagnosis and treatment of acute and chronic heart failure of the European Society of Cardiology (ESC). Developed with the special contribution of the Heart
Failure Association (HFA) of the ESC. Eur J Heart Fail. 2016 Aug;18(8):891-975.

13 Authors/Task Force Members, Piepoli MF, Hoes AW, Agewall S, Albus C, Brotons C, Catapano AL, et al. 2016 European Guidelines on cardiovascular disease prevention in clinical practice: the Sixth Joint Task Force of the European Society of Cardiology and Other Societies on Cardiovascular Disease Prevention in Clinical Practice (constituted by representatives of 10 societies and by invited experts): developed with the special contribution of the European Association for Cardiovascular Prevention \& Rehabilitation (EACPR). Eur J Prev Cardiol. 2016 Jul; 23(11):NP1-96.

14 Seferovic PM, Ponikowski P, Anker SD, Bauersachs J, Chioncel O, Cleland JG, et al. Clinical practice update on heart failure 2019: pharmacotherapy, procedures, devices and patient management. An expert consensus meeting report of the Heart Failure Association of the European Society of Cardiology. Eur J Heart Fail. 2019 Oct;21(10): 1169-86.

15 Davies MJ, D’Alessio DA, Fradkin J, Kernan WN, Mathieu C, Mingrone G, et al. Management of hyperglycaemia in type 2 diabetes, 2018. A consensus report by the American Diabetes Association (ADA) and the European Association for the Study of Diabetes (EASD). Diabetologia. 2018 Dec;61(12): 2461-98.

16 McMurray JJ, Solomon SD, Inzucchi SE Køber L, Kosiborod MN, Martinez FA, et al.; DAPA-HF Trial Committees and Investigators. Dapagliflozin in Patients with Heart Failure and Reduced Ejection Fraction. N Engl J Med. 2019 Nov;381(21):1995-2008. 